\title{
The gap probabilities of the tacnode, Pearcey and Airy point processes, their mutual relationship and evaluation
}

\author{
M. Bertola ${ }^{\dagger \dagger 1}$, M. Cafasso $\diamond 2$ \\ † Centre de recherches mathématiques, Université de Montréal \\ C. P. 6128, succ. centre ville, Montréal, Québec, Canada H3C 3J7 \\ ¥Department of Mathematics and Statistics, Concordia University \\ 1455 de Maisonneuve W., Montréal, Québec, Canada H3G 1 M8 \\ $\diamond$ LUNAM Université, LAREMA, Université d'Angers \\ 2 Boulevard Lavoisier, 49045 Angers, France.
}

\begin{abstract}
We express the gap probabilities of the tacnode process as the ratio of two Fredholm determinants; the denominator is the standard Tracy-Widom distribution, while the numerator is the Fredholm determinant of a very explicit kernel constructed with Airy functions and exponentials. The formula allows us to apply the theory of numerical evaluation of Fredholm determinants and thus produce numerical results for the gap probabilities. In particular we investigate numerically how, in different regimes, the Pearcey process degenerates to the Airy one, and the tacnode degenerates to the Pearcey and Airy ones.
\end{abstract}

\section{Introduction}

The study of "infinite-dimensional diffusions" arising from the scaling limit of determinantal point processes attracted much attention in the last years, see for instance [4], chapters 6,10,11,38 and references therein. One of the most popular model has been introduced by Dyson in [11], where a dynamical version of the probability distributions arising in random matrix theory is defined. The idea is to study $N$ Brownian particles moving on the real line, conditioned not to intersect. When the starting and ending points are fixed and $N$ goes to infinity, the particles sweep out a certain region in space-time, whose shape depends on the number and relative position of the starting and ending points. Interesting infinite dimensional diffusions arise when studying the behavior of the particles near the boundary of this region. Near the points where this boundary is a smooth curve, the fluctuations of the particles are described by the well known Airy process [19, 14, 15]. Near a cusp singularity we are lead to the study of the Pearcey process $[24,18]$. The most recently studied case is the one of the so-called tacnode singularity, appearing when the boundary looks

\footnotetext{
${ }^{1}$ bertola@crm.umontreal.ca

2 cafasso@math.univ-angers.fr
} 
(locally) as two circles touching at one point. The first expression for the kernel of the tacnode process has been written in [2], as a scaling limit of a model of random walks, continuous in time. Few months later, in [10], the authors found a different expression (for the one-time case) in terms of a $4 \times 4$ Riemann-Hilbert problem, this time starting from non-intersecting Brownian motions. Another formula for the multi-time case, again different from the previous ones, has been found by Johansson in [16]. There, for the first time, the resolvent of the Airy kernel appeared in the formula of the tacnode kernel (this is quite an important point for our present work, as it will be apparent later). In [3], finally, the authors analyzed the same process as arising from random tilings instead of Dyson Brownian motions: in this paper it has been proven that all the different formulations above are indeed equivalent, thus performing a significative step in the direction of universality for the tacnode process. A similar result has been obtained by Delvaux in [9]. A more general formulation of this process in the "asymmetric" case has been studied in [12].

While the kernels describing the Airy and Pearcey processes can be expressed as simple double integrals of exponential functions (both in the one and multi-time case), studying the tacnode process presents an additional complication, since its kernel is highly transcendental. Indeed, it is written in terms of the resolvent of the Airy operator. In particular, given the known representations of the tacnode kernel, the study of the associated (joint) gap probability (i.e. the Fredholm determinant associated to the kernel) appears as quite complicated.

The purpose of this short investigation is to show how to express the gap probability of the tacnode process in terms of Fredholm determinants associated to explicit kernels, no more complicated than the standard Airy one. Explicitly the key theoretical result is Theorem 3.2 that expresses the gap probability of the tacnode process as a ratio of determinants (notation to be defined therein)

$$
\operatorname{Prob}\left\{\mathcal{T}_{\sigma}\left(\tau_{i}\right) \notin E^{(i)}, \quad i=1, \ldots, r\right\}=\frac{\operatorname{det}\left[\operatorname{Id}-\mathbb{H}_{E}\right]}{\operatorname{det}\left[\operatorname{Id}_{[\widetilde{\sigma}, \infty)}-\pi_{\widetilde{\sigma}} K_{\mathrm{Ai}} \pi_{\widetilde{\sigma}}\right]}
$$

where $\mathcal{T}_{\sigma}(\tau)$ denotes the tacnode point field at time $\tau$, the operator $\mathbb{H}$ acts on

$$
L^{2}\left(\mathbb{R}_{+} \sqcup \mathbb{R}_{0} \bigsqcup_{j=1}^{r} \mathbb{R}_{\tau_{j}}\right) \simeq L^{2}\left(\mathbb{R}_{+}\right) \oplus L^{2}\left(\mathbb{R}_{0}\right) \bigoplus_{j=1}^{r} L^{2}\left(\mathbb{R}_{\tau_{j}}\right)
$$

and the subscript ${ }_{E}$ means that it is restricted to the following subspace

$$
L^{2}\left(\mathbb{R}_{+}\right) \oplus L^{2}([\widetilde{\sigma}, \infty)) \oplus L^{2}\left(E^{(1)} \sqcup \ldots \sqcup E^{(r)}\right) .
$$

In the formula above, the subscripts on the various copies of $\mathbb{R}$ are simply to distinguish them from each other and remind that some are associated to the times $\tau_{1}, \ldots, \tau_{r}$ and each $E^{(j)}, j=1, \ldots, r$, is a finite union of bounded intervals. The kernel $\mathbb{H}$ is described explicitly in Theorem 3.2, but here it suffices to say that it involves at most the standard Airy functions. In the one-time case it is a $3 \times 3$ kernel acting on $L^{2}\left(\mathbb{R}_{+}\right) \oplus L^{2}(\mathbb{R}) \oplus L^{2}(\mathbb{R})$ and reading as follows:

$$
\mathbb{H}(x, y)=\left[\begin{array}{c|c|c}
0 & -\mathrm{Ai}(x+y) & \mathrm{Ai}^{(-\tau)}(x \sqrt[3]{2}+\sigma-y) \\
\hline-\operatorname{Ai}(x+y) & 0 & \mathrm{Ai}^{(-\tau)}(x \sqrt[3]{2}+y-\sigma) \\
\hline \mathrm{Ai}^{(\tau)}(\sigma-x+y \sqrt[3]{2}) & \mathrm{Ai}^{(\tau)}(x-\sigma+y \sqrt[3]{2}) & 0
\end{array}\right]
$$


where $\operatorname{Ai}^{(\tau)}(x)=2^{\frac{1}{6}} \mathrm{e}^{\tau x+\frac{2}{3} \tau^{3}} \mathrm{Ai}\left(x+\tau^{2}\right)$ and $\tau, \sigma$ are parameters of the tacnode process, respectively the time and the "pressure" (or overlap, see also below for details). As an application of our result, we compute numerically, following the method of [8], the gap probability associated to the tacnode process, and its relationship with the Airy and Pearcey processes (a more detailed list of our results is given below). Theorem 3.2 can also be used as a starting point to identify the tacnode gap probability with the tau function of a given Riemann-Hilbert problem, in the spirit of $[6,5]$. A work in this direction is currently in preparation [7].

Before entering into the details of our results, let us spend few words about Fredholm determinants and their numerical evaluations. Let $(X, \mathrm{~d} \mu)$ be a (sigma-finite) measure space. Given an integral operator $\mathcal{J}$ on $L^{2}(X, \mathrm{~d} \mu)$ (belonging to some trace ideal [20]) the computation of the (regularized) Fredholm-Carleman determinant is an essentially transcendental problem. If the kernel $\mathbb{J}$ of the integral operator $\mathcal{J}$ is known and sufficiently regular, then the numerical evaluation of the determinant can be approached by a suitable approximation scheme which involves projecting onto a suitable finite dimensional subspace. In this way the Fredholm determinant can be approximated by a finite determinant.

The numerical evaluation of Fredholm determinants of operators on $\mathbb{R}$ (or disjoint unions of several copies thereof) has been recently shown to pose little obstacle [8]; the gist of the beautiful idea is basically the simplest approach of discretization, paired with skillful use of numerical estimates associated with quadrature rules.

The effectiveness of the method is vastly improved if the kernel is explicit in terms of functions that are already implemented or easily so. The paramount example is the Airy kernel and the corresponding Tracy-Widom [23] distribution

$$
\begin{array}{r}
K_{\mathrm{Ai}}(x, y):=\frac{\operatorname{Ai}(x) \operatorname{Ai}^{\prime}(y)-\operatorname{Ai}^{\prime}(x) \operatorname{Ai}(y)}{x-y} F_{2}(s):=\operatorname{det}\left[\operatorname{Id}_{[s, \infty)}-\pi_{s} K_{\mathrm{Ai}} \pi_{s}\right], \\
\pi_{s}: L^{2}(\mathbb{R}) \rightarrow L^{2}([s, \infty) .
\end{array}
$$

As we said before, the kernel of the tacnode process is, however, highly transcendental; it is precisely with the results contained in Theorem 3.2 that we make it directly amenable to the methods in [8].

The following sections are organized as follows:

- In the second section, as a sort of warm-up, we show how to use the theoretical results in [6] to compute (numerically) the gap probability of the Pearcey process. As an application, we give numerical confirmations of the degeneration of the Pearcey gap probability to a couple of Tracy-Widom distribution (see [6] and, for a similar result, [1]).

- In the third section, we start with our main theoretical result, i.e. the expression of the tacnode gap probability in terms of the ratio of two Fredholm determinants with simpler kernels. This allows us to show numerically, in the two subsections, how the tacnode gap probability degenerate, in different regimes, to the Pearcey and the Airy one. The degeneration tacnodeto-Pearcey has been already proven in [13] and another proof will be given in [7], together with the tacnode-to-Airy one, which has not been proven yet (to the best of our knowledge). 
- In the appendix we prove that, given a determinantal point process and a given subset of the state space, the point process conditioned not to have particles on that given subset is also determinantal, and we explicitly write its kernel (see also [22] for the case of point processes with a finite number of particles, and [17] for the discrete case). In the third section we will relate (formally) this result to our representation of the tacnode gap probability as a ratio of two Fredholm determinants.

\section{Numerical evaluation of the Pearcey gap probabilities}

Suppose that $I=\left[a_{1}, a_{2}\right] \cup \ldots \cup\left[a_{2 N-1}, a_{2 N}\right]$ and denote with $\mathcal{P}$ the Pearcey process $[24,18]$; in $[6]$ it was proved that

$$
\mathbb{P}\{\mathcal{P}(\tau) \notin I\}=\operatorname{det}\left[\mathbf{1}-\widetilde{K}_{\vec{a}}\right]
$$

where the Fredholm determinant on the right hand side is on the space $L^{2}\left(\gamma_{L} \cup i \mathbb{R} \cup \gamma_{R}\right)$ and the operator $\widetilde{K}$ has kernel $\widetilde{K}(\lambda, \mu)$ given by (here $\chi_{X}$ denotes the indicator function of a subset $X \subset \mathbb{C}$ )

$$
\begin{aligned}
& \widetilde{K}_{\vec{a}}(\lambda, \mu)=\frac{1}{2 i \pi} \frac{\mathrm{e}^{\frac{\Theta_{0}(\lambda)-\Theta_{0}(\mu)}{2}} \chi_{i \mathbb{R}}(\mu) \chi_{\gamma_{R} \cup \gamma_{L}}(\lambda)-\sum_{j=1}^{2 N}(-)^{j} \mathrm{e}^{\frac{\Theta_{0}(\mu)-\Theta_{0}(\lambda)}{2}+a_{j} \lambda-a_{j} \mu} \chi_{\gamma_{R} \cup \gamma_{L}}(\mu) \chi_{i \mathbb{R}}(\lambda)}{\lambda-\mu} \\
& \Theta_{0}(\lambda):=\frac{\lambda^{4}}{4}-\tau \frac{\lambda^{2}}{2}
\end{aligned}
$$

The contours $\gamma_{R}$ is a contour in the right halfplane that comes from infinity along $\arg \lambda=\frac{\pi}{4}$ and returns to infinity along $\arg \lambda=-\frac{\pi}{4}$ while $\gamma_{L}=-\gamma_{R}$. Our aim is to use the equivalent representation of the Percey gap probability given in (2.2) for numerical computations. For the case of multi-time Pearcey gap probabilities we refer to $[5]^{3}$. In order to apply Bornemann's procedure [8] we prefer to have kernels acting on the interval $[0,1]$, hence we have chosen two hyperbolas parametrized by

$$
\begin{array}{r}
\phi_{R}(s)=\frac{1}{2}\left[\frac{s}{1-s}+\frac{1-s}{s}\right]-\frac{i}{2}\left[\frac{s}{1-s}-\frac{1-s}{s}\right]:(0,1) \rightarrow \gamma_{R} \\
\phi_{L}(s)=-\frac{1}{2}\left[\frac{s}{1-s}+\frac{1-s}{s}\right]+\frac{i}{2}\left[\frac{s}{1-s}-\frac{1-s}{s}\right]:(0,1) \rightarrow \gamma_{L} \\
\phi_{0}(s)=\tan \left(\frac{2 \pi s-\pi}{2}\right):(0,1) \rightarrow i \mathbb{R}
\end{array}
$$

and, pulling back the kernel $\widetilde{K}$ with the aid of the maps $\phi_{L, R, 0}$, we obtained a kernel on $(0,1)^{3}$ (it can be extended to the closure by defining it as zero). Then the numerical evaluation of the resulting kernel can be carried out as in [8]. We only point out that the Pearcey kernel involves special functions (Pearcey integrals) that are not implemented in any library that we could find. Thus it seems that the equivalent representation (2.1) is convenient not only from the theoretical point of view to analyze the transition to the Airy process as in [6], but also from the point of view of effective numerical implementation.

\footnotetext{
${ }^{3}$ We remark that in the cited reference the formulæ $(3.11,3.12)$ should have an additional overall denominator $2 i \pi$ and in the last line of formula $(3.12)$ the alternating sign $(-1)^{\ell}$ should be $(-1)^{\ell+1}$. Similarly, the formulæ $(2.12$, $2.13,2.14)$ should all have an additional overall factor $\frac{1}{2 i \pi}$.
} 

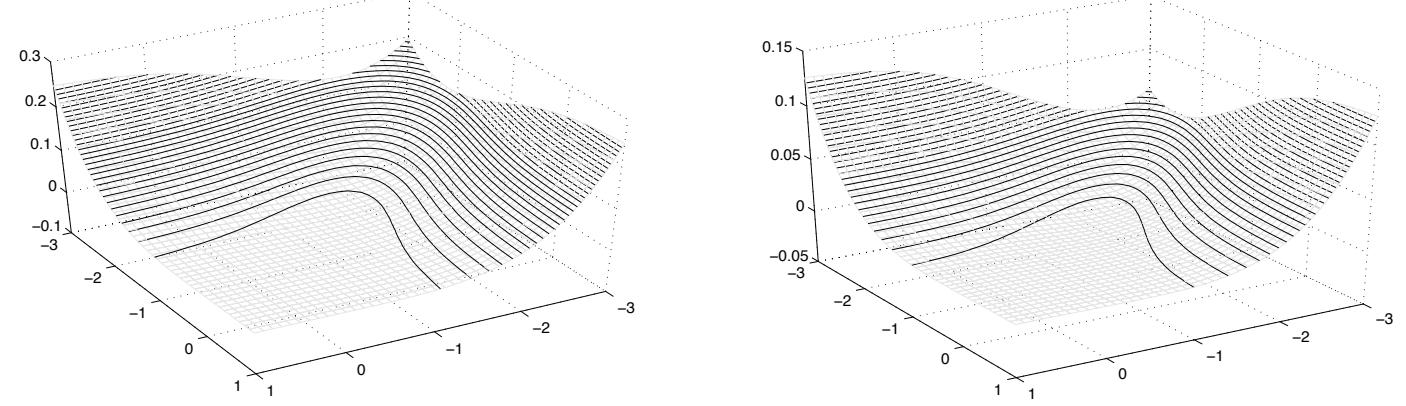

Figure 1: The relative difference $1-\frac{F_{\mathrm{P}}\left(\left[a_{P}, b_{P}\right] ; \tau\right)}{F_{2}(\sigma) F_{2}(\rho)}$ with the parameters as in $(2.8),(\rho, \sigma) \in[-3,1] \times[-3,1]$ and $\tau=3$ (left) or $\tau=5.314$ (right).

\subsection{From the Pearcey to the Airy process}

Let us consider, for instance, the simplest gap probabilities for the Pearcey and Airy processes:

$$
F_{\mathrm{P}}\left(\left[a_{p}, b_{p}\right] ; \tau\right)=\mathbb{P}\left\{\mathcal{P}(\tau) \notin\left[a_{p}, b_{p}\right]\right\}, \quad F_{2}([\sigma, \infty))=\mathbb{P}\{\mathcal{A} \notin[\sigma, \infty)\}
$$

In [6] it was shown by using the Deift-Zhou nonlinear steepest descent method that, in particular

$$
F_{\mathrm{P}}\left(\left[-2\left(\frac{\tau}{3}\right)^{\frac{3}{2}}+(3 \tau)^{\frac{1}{6}} \rho, 2\left(\frac{\tau}{3}\right)^{\frac{3}{2}}-(3 \tau)^{\frac{1}{6}} \sigma,\right] ; \tau\right) \underset{\tau \rightarrow \infty}{\longrightarrow} F_{2}([\sigma, \infty)) F_{2}([\rho, \infty))
$$

(the statement of [6] was for an arbitrary finite union of bounded intervals, but here we restrict to the simplest nontrivial example). As an indirect confirmation that the numerical approach is sound, we verify this limit numerically plotting the graph of the "relative difference" $1-\frac{F_{P}\left(\left[a_{P}, b_{P}\right] ; \tau\right)}{F_{2}(\sigma) F_{2}(\rho)}$ with the parameters as in $(2.8)$ and $(\rho, \sigma) \in[-3,1] \times[-3,1]$. The two graphs in Figure 1 correspond to the case $\tau=3$ and $\tau=5.314$. In the second case, already for a relatively small value of $\tau$, the relative difference is below $15 \%$ (in the range of space variables we considered).

\section{Gap probabilities of the tacnode process as ratio of deter- minants}

We start recalling one of the three equivalent formulations of the tacnode kernel obtained in [3]:

Definition 3.1 ([3] formula (19)) The kernel of the extended tacnode process with overlap $\sigma$ is defined as

$$
\begin{aligned}
\mathbb{K}^{\operatorname{tac}}\left(\tau_{1}, \xi_{1} ; \tau_{2}, \xi_{2}\right)=K_{\mathrm{Ai}}^{\left(\tau_{1},-\tau_{2}\right)} & \left(\sigma-\xi_{1}, \sigma-\xi_{2}\right)-\operatorname{Id}_{\tau_{1}>\tau_{2}} p\left(\tau_{1}-\tau_{2} ; \xi_{1}, \xi_{2}\right)+ \\
& +\int_{\tilde{\sigma}}^{\infty}\left(\left(\mathbf{1}-K_{\mathrm{Ai}}\right)_{\tilde{\sigma}}^{-1} \mathcal{A}_{\xi_{1}-\sigma}^{\tau_{1}}\right)(u) \mathcal{A}_{\xi_{2}-\sigma}^{-\tau_{2}}(u) d u
\end{aligned}
$$


where $\widetilde{\sigma}=2^{\frac{2}{3}} \sigma$ and $K_{\mathrm{Ai}}$ denotes the usual Airy kernel and ${ }^{4}$

$$
\begin{aligned}
p\left(\Delta \tau ; \xi_{1}, \xi_{2}\right) & :=\frac{e^{-\frac{\left(\xi_{1}-\xi_{2}\right)^{2}}{4 \Delta \tau}}}{\sqrt{4 \pi \Delta \tau}} \\
\operatorname{Ai}^{(\tau)}(x) & :=\frac{2^{\frac{1}{6}}}{2 \pi i} \int_{\gamma_{R}} d z e^{z^{3} / 3+z^{2} \tau-z x}=2^{\frac{1}{6}} e^{\tau x+\frac{2}{3} \tau^{3}} \operatorname{Ai}\left(x+\tau^{2}\right), \\
K_{\mathrm{Ai}}^{\left(\tau_{1},-\tau_{2}\right)}(x, y) & :=\int_{0}^{\infty} \operatorname{Ai}^{\left(\tau_{1}\right)}(x+u \sqrt[3]{2}) \mathrm{Ai}^{\left(-\tau_{2}\right)}(y+u \sqrt[3]{2}) d u, \\
\mathcal{A}_{\xi}^{\tau}(u) & :=\operatorname{Ai}^{(\tau)}\left(\xi+2^{1 / 3} u\right)-\int_{0}^{\infty} \operatorname{Ai}^{(\tau)}\left(-\xi+2^{1 / 3} v\right) \operatorname{Ai}(u+v) d v,
\end{aligned}
$$

Given $r$ times $\tau_{1}<\tau_{2}<\ldots<\tau_{r}$ we associate to each a copy of $\mathbb{R}$ or, equivalently we consider the Cartesian product $\mathbb{R} \times\left\{\tau_{1}, \ldots, \tau_{r}\right\}$ which is set-theoretically isomorphic to $\mathbb{R}^{r}$. Another convenient way of thinking about this set is as the disjoint union of $\mathbb{R}$ with itself, $r$ times. We will use the notation $\mathbb{R}_{\tau_{j}}$ to refer to the $j$-th copy (which should be thought of as a copy of $\mathbb{R}$ "at time $\tau_{j}$ "). In each copy $\mathbb{R}_{\tau_{j}}$ we consider a Borel bounded subset $E^{(j)}$; although it is much less general, the reader should imagine that each $E^{(j)}$ is a finite union of bounded intervals as follows

$$
E^{(j)}=\left[a_{1}^{(j)}, b_{1}^{(j)}\right] \cup \ldots \cup\left[a_{\ell_{j}}^{(j)}, b_{\ell_{j}}^{(j)}\right], \quad a_{1}^{(j)}<b_{1}^{(j)}<a_{2}^{(j)}<b_{2}^{(j)}<\ldots<a_{\ell_{j}}^{(j)}<b_{\ell_{j}}^{(j)} .
$$

We denote by $\mathcal{T}_{\sigma}(\tau)$ the tacnode field at time $\tau$; the joint gap probability of the multi-set $E:=$ $E^{(1)} \sqcup \ldots \sqcup E^{(r)}$ is the probability that there are no points of the tacnode process in $E^{(j)}$ at time $\tau_{j}$, for all $j=1, \ldots, r$ :

$$
F_{\mathrm{tac}}(E, \vec{\tau} ; \sigma):=\operatorname{Prob}\left\{\mathcal{T}_{\sigma}\left(\tau_{j}\right) \notin E^{(j)}, \quad j=1, \ldots, r\right\} .
$$

Then the general theory of determinantal random point processes states that

$$
F_{\mathrm{tac}}(E, \vec{\tau} ; \sigma)=\operatorname{det}\left(\mathbf{1}-\left.\mathbb{K}^{\mathrm{tac}}\right|_{E}\right)
$$

where the operator $\mathbb{K}^{\text {tac }}$ is intended as the operator on $L^{2}\left(\mathbb{R}_{\tau_{1}} \sqcup \ldots \sqcup \mathbb{R}_{\tau_{r}}\right)$ with kernel as in Definition 3.1. The main goal is to express the determinant (3.8) as the ratio of two simpler Fredholm determinants. The following two theorems, nevertheless, will be stated for the (more general) generating functions of the occupation numbers (see [21] for details); if $E^{(i)}$ is a disjoint union of intervals $E^{(j)}=\bigsqcup_{\alpha=1}^{\ell_{j}} E_{\alpha}^{(j)}$, this latter is defined by

$$
F_{\mathrm{tac}}(E, \vec{\tau} ; \sigma, \vec{z}):=\left\langle\prod_{j=1}^{r} \prod_{\alpha=1}^{\ell_{j}}\left(z_{\alpha}^{(j)}\right)^{\sharp_{E_{\alpha}^{(j)}}}\right\rangle=\operatorname{det}\left[\mathbf{1}-\left(\sum_{j=1}^{r} \sum_{\alpha=1}^{\ell_{j}}\left(1-z_{\alpha}^{(j)}\right) \Pi_{E_{\alpha}^{(j)}}\right) \mathbb{K}^{\mathrm{tac}} \vec{\Pi}\right]
$$

where $\vec{\Pi}=\sum_{j=1}^{r} \sum_{\alpha=1}^{\ell_{j}} \Pi_{E_{\alpha}^{(j)}}$ is the projector on the multi-interval $\vec{E}$ and the generating function is a series on the variables $z_{\alpha}^{(j)}$. The gap probability is nothing but the value of the generating function at $z_{\alpha}^{(j)}=0$. We shall use the notations

$$
\Pi_{z}^{(j)}:=\sum_{\alpha=1}^{\ell_{j}}\left(1-z_{\alpha}^{(j)}\right) \Pi_{E_{\alpha}^{(j)}}, \quad \vec{\Pi}_{z}:=\sum_{j=1}^{r} \Pi_{z}^{(j)} .
$$

\footnotetext{
${ }^{4}$ For our convenience we slightly modified the definition of $\mathrm{Ai}^{(\tau)}$ multiplying it by the coefficient $2^{\frac{1}{6}}$. Nevertheless formulas (3.1), (3.4) and (3.5) are changed accordingly so that the kernel is truly the same as in [3].
} 
Theorem 3.1 The generating function (3.9) admits the equivalent representation

$$
F_{\mathrm{tac}}(E, \tau ; \sigma, \vec{z})=F_{2}(\widetilde{\sigma})^{-1} \operatorname{det}\left(\mathbf{1}-\left[\begin{array}{c|ccc}
\pi K_{\mathrm{Ai}} \pi & -\pi \mathfrak{A}_{-\tau_{1}}^{T} \Pi^{(1)} & \ldots & -\pi \mathfrak{A}_{-\tau_{r}}^{T} \Pi^{(r)} \\
\hline-\Pi_{z}^{(1)} \mathfrak{A}_{\tau_{1}} \pi & & & \\
\vdots & & \vec{\Pi}_{z}\left(\mathbb{K}_{0}-\mathbb{G}\right) \vec{\Pi} & \\
-\Pi_{z}^{(r)} \mathfrak{A}_{\tau_{r}} \pi & &
\end{array}\right]\right)
$$

where $F_{2}(\widetilde{\sigma})$ is the Tracy-Widom distribution (1.3) and $\mathbb{K}_{0}, \mathbb{G}: L^{2}\left(\mathbb{R}_{\tau_{1}} \sqcup \ldots \sqcup \mathbb{R}_{\tau_{r}}\right) \rightarrow L^{2}\left(\mathbb{R}_{\tau_{1}} \sqcup \ldots \sqcup \mathbb{R}_{\tau_{r}}\right)$ are the operators with kernels

$$
\left(\mathbb{K}_{0}\right)_{i j}\left(\xi_{1}, \xi_{2}\right)=K_{\mathrm{Ai}}^{\left(\tau_{i},-\tau_{j}\right)}\left(\sigma-\xi_{1}, \sigma-\xi_{2}\right), \quad \mathbb{G}_{i j}\left(\xi_{1}, \xi_{2}\right)=\mathbf{1}_{\tau_{1}>\tau_{2}} \frac{e^{-\frac{\left(\xi_{1}-\xi_{2}\right)^{2}}{4\left(\tau_{1}-\tau_{2}\right)}}}{\sqrt{4 \pi\left(\tau_{1}-\tau_{2}\right)}}
$$

while $\mathfrak{A}_{\vec{\tau}}: L^{2}(\mathbb{R}) \rightarrow L^{2}\left(\mathbb{R}_{\tau_{1}} \sqcup \ldots \sqcup \mathbb{R}_{\tau_{r}}\right)$ has kernel

$$
\left[\mathfrak{A}_{\vec{\tau}}(x, u)\right]_{j}=\operatorname{Ai}^{\left(\tau_{j}\right)}\left(x-\sigma+2^{1 / 3} u\right)-\int_{0}^{\infty} \operatorname{Ai}^{\left(\tau_{j}\right)}\left(\sigma-x+2^{1 / 3} v\right) \operatorname{Ai}(v+u) d v .
$$

Proof. With the notations introduced above, the tacnode kernel defines an operator on $L^{2}\left(\mathbb{R}_{\tau_{1}} \sqcup \ldots \sqcup \mathbb{R}_{\tau_{r}}\right)$ that equals to $\left[\mathbb{K}_{0}-\mathbb{G}_{i j}+\mathfrak{A}_{\tau_{i}}\left(\mathbf{1}-\pi K_{\mathrm{Ai}} \pi\right)^{-1} \mathfrak{A}_{-\tau_{j}}^{T}\right.$.

The identity is based on the following operator identity (all being trace-class perturbations of the identity)

$$
\begin{aligned}
& \operatorname{det}\left(\mathbf{1}-\left[\begin{array}{c|c}
\pi K_{\mathrm{Ai}} \pi & -\pi \mathfrak{A}_{-\vec{\tau}}^{T} \vec{\Pi} \\
\hline-\vec{\Pi}_{z} \mathfrak{A}_{\vec{\tau}} \pi & \vec{\Pi}_{z}\left(\mathbb{K}_{0}-\mathbb{G}\right) \vec{\Pi}
\end{array}\right]\right)= \\
& =\operatorname{det}\left[\begin{array}{c|c|c}
\mathbf{1}-\pi K_{\mathrm{Ai}} \pi & 0 \\
\hline 0 & \mathbf{1}
\end{array}\right] \operatorname{det}\left[\begin{array}{c|c}
\mathbf{1} & 0 \\
\hline \vec{\Pi}_{z} \mathfrak{A}_{\vec{\tau}} \pi & \mathbf{1}
\end{array}\right] \operatorname{det}\left[\begin{array}{c|c}
\mathbf{1} & \left(\mathbf{1}-\pi K_{\mathrm{Ai}} \pi\right)^{-1} \mathfrak{A}_{-\vec{\tau}}^{T} \vec{\Pi} \\
\hline 0 & \mathbf{1}-\vec{\Pi}_{z}\left(\mathbb{K}_{0}-\mathbb{G}_{\vec{\tau}}+\mathfrak{A}_{\vec{\tau}}\left(\mathbf{1}-\pi K_{\mathrm{Ai}} \pi\right)^{-1} \mathfrak{A}_{-\vec{\tau}}^{T}\right) \vec{\Pi}
\end{array}\right]= \\
& =\underbrace{\operatorname{det}\left(\mathbf{1}-\pi K_{\mathrm{Ai}} \pi\right)}_{F_{2}(\widetilde{\sigma})} \operatorname{det}\left(\mathbf{1}-\vec{\Pi}_{z} \mathbb{K}^{\operatorname{tac}} \vec{\Pi}\right)
\end{aligned}
$$

\section{Q.E.D}

Remark 3.1 [Tacnode gap probabilities as (formal) conditioned process] The gap probabilities of the tacnode process are the ratio of two Fredholm determinants: the denominator is the TracyWidom distribution, i.e., the gap probability of the Airy process. It is enticing to interpret thus the ratio as a conditional probability. For this interpretation to hold the numerator of formula (3.11) should be a gap probability of a determinantal process on the configuration space $\mathbb{R}_{0} \sqcup\left(\bigsqcup_{j=1}^{r} \mathbb{R}_{\tau_{j}}\right)$ with kernel as indicated in the formula itself.

Then the tacnode would be the conditioned process where the conditioning is that there are no points in $[\widetilde{\sigma}, \infty) \subset \mathbb{R}_{0}$. This type of of conditioned processes have been already analyzed in [17] for the discrete case, and in [22] for the continuous case with a finite number of particles. In Appendix A we treat the continuous case with an arbitrary number of particles and give an explicit expression for the kernel of the conditioned process. However, the correlation functions of this "extended" tacnode process fail the positivity test (we checked on some numerical example) and thus the interpretation is only formal. 
The second theorem is an equivalent representation where the operator in the Fredholm determinant in the numerator of Theorem 3.1 is further "unraveled" in terms of elementary terms.

Theorem 3.2 With the same notations as in Theorem 3.1 we have

$$
F_{\mathrm{tac}}(E, \vec{\tau} ; \sigma, \vec{z})=\frac{\operatorname{det}\left[\mathbf{1}-\widehat{\Pi}_{z} \mathbb{H} \widehat{\Pi}\right]}{\operatorname{det}\left[\mathbf{1}-\pi K_{\mathrm{Ai}} \pi\right]}
$$

where the Fredholm determinant is defined on the space

$$
L^{2}\left(\mathbb{R}_{+} \sqcup \mathbb{R}_{0} \sqcup \mathbb{R}_{\tau_{1}} \sqcup \ldots, \sqcup \mathbb{R}_{\tau_{r}}\right) \simeq L^{2}\left(\mathbb{R}_{+}\right) \oplus L^{2}\left(\mathbb{R}_{0}\right) \oplus\left(\mathbb{R}_{\tau_{1}} \sqcup \ldots \sqcup \mathbb{R}_{\tau_{r}}\right) .
$$

Denoting the $L^{2}\left(\mathbb{R}_{+}\right)$with the index -1 , the operators that appear are the following ones

$$
\widehat{\Pi}_{z}:=\mathbf{1}_{-1} \oplus \pi \oplus \vec{\Pi}_{z}, \quad \widehat{\Pi}=\widehat{\Pi}_{0}=\operatorname{Id}_{-1} \oplus \pi \oplus \vec{\Pi}
$$

and their kernel $\mathbb{H}$ is expressed in terms of ordinary Airy functions as follows

$\left[\begin{array}{c|c|c}\mathbb{H}_{-1,-1} \equiv 0 & \mathbb{H}_{-1,0}(x, y)=-\mathrm{Ai}(x+y) & \mathbb{H}_{-1, j}(x, y)=\mathrm{Ai}^{\left(-\tau_{j}\right)}(x \sqrt[3]{2}+\sigma-y) \\ \hline \mathbb{H}_{0,-1}(x, y)=-\mathrm{Ai}(x+y) & \mathbb{H}_{0,0} \equiv 0 & \mathbb{H}_{0, j}(x, y)=\mathrm{Ai}^{\left(-\tau_{j}\right)}(x \sqrt[3]{2}+y-\sigma) \\ \hline \mathbb{H}_{i,-1}(x, y)=\mathrm{Ai}^{\left(\tau_{i}\right)}(\sigma-x+y \sqrt[3]{2}) & \mathbb{H}_{i, 0}(x, y)=\mathrm{Ai}^{\left(\tau_{i}\right)}(x-\sigma+y \sqrt[3]{2}) & \mathbb{H}_{i, j}(x, y)=-p\left(\tau_{i}-\tau_{j} ; x, y\right) \chi_{i>j}\end{array}\right]$

with $1 \leq i, j \leq r$.

The expression of the Fredholm determinant in Theorem 3.2 is the most suitable for the numerical computations because each entry of the kernel is either a simple modification of the Airy function or exponential. The gap probabilities are obtained simply by setting all the $z_{\alpha}^{(j)}$ 's to zero, or -which is the same- restricting the operator $\mathbb{H}$ on the set $\mathbb{R}_{+} \sqcup[\widetilde{\sigma}, \infty) \sqcup\left(\bigsqcup_{j=1}^{R} E^{(j)}\right)$.

Proof of Theorem 3.2. For brevity we shall introduce the operators with the kernels below

$$
\begin{aligned}
& \mathcal{C}_{\tau}(\xi, u):=2^{\frac{1}{6}} \mathrm{Ai}^{(\tau)}(\xi-\sigma+u \sqrt[3]{2}): L^{2}\left(\mathbb{R}_{+}\right) \rightarrow L^{2}\left(\mathbb{R}_{\tau}\right) \\
& \mathcal{C}(u, v):=\operatorname{Ai}(u+v): L^{2}\left(\mathbb{R}_{+}\right) \rightarrow L^{2}(\mathbb{R})
\end{aligned}
$$

The first observation is that both $K_{\mathrm{Ai}}$ and $K_{\mathrm{Ai}}^{\left(\tau_{1},-\tau_{2}\right)}$ can be written as compositions;

$$
K_{\mathrm{Ai}}=\mathcal{C C}^{T}, \quad K_{\mathrm{Ai}}^{\left(\tau_{1},-\tau_{2}\right)}=\mathcal{C}_{\tau_{1}} \mathcal{C}_{-\tau_{2}}^{T}
$$

If we denote the parity operator around $\sigma$ by $P: L^{2}(\mathbb{R}) \rightarrow L^{2}(\mathbb{R})(P f)(x)=f(2 \sigma-x)$ then the tacnode kernel can be written as

$$
\mathbb{K}_{i j}^{\mathrm{tac}}=P \mathcal{C}_{\tau_{i}} \mathcal{C}_{-\tau_{j}}^{T} P+\mathfrak{A}_{\tau_{i}} \pi\left(\mathbf{1}-\pi \mathcal{C} \mathcal{C}^{T} \pi\right)^{-1} \pi \mathfrak{A}_{-\tau_{j}}^{T}-\mathbb{G}_{i j}
$$


where

$$
\left[\mathfrak{U}_{\tau}\right]_{j}=\mathcal{C}_{\tau_{j}}-P \mathcal{C}_{\tau_{j}} \mathcal{C}: L^{2}\left(\mathbb{R}_{+}\right) \rightarrow L^{2}\left(\mathbb{R}_{\tau_{j}}\right)
$$

We denote $\mathcal{C}_{\vec{\tau}}: L^{2}\left(\mathbb{R}_{+}\right) \rightarrow L^{2}\left(\bigsqcup_{j=1}^{r} \mathbb{R}_{\tau_{j}}\right) \simeq \bigoplus_{j=1}^{r} L^{2}\left(\mathbb{R}_{\tau_{j}}\right)$ as the operator with components $\mathcal{C}_{\tau_{j}}$; then the proof relies upon the following identity of determinants

$$
\operatorname{det}\left(\mathbf{1}-\left[\begin{array}{c|c}
\pi \mathcal{C} \mathcal{C}^{T} \pi & -\pi \mathfrak{A}_{-\vec{\tau}}^{T} \vec{\Pi} \\
\hline-\vec{\Pi}_{z} \mathfrak{A}_{\vec{\tau}} \pi & \vec{\Pi}_{z}\left(\mathbb{K}_{0}-\vec{G}\right) \vec{\Pi}
\end{array}\right]\right)=\operatorname{det}\left[\begin{array}{c|c|c}
\mathbf{1} & \mathcal{C}^{T} \pi & -\mathcal{C}_{-\vec{\tau}}^{T} P \vec{\Pi} \\
\hline \pi \mathcal{C} & \mathbf{1} & -\pi \mathcal{C}_{-\vec{\tau}}^{T} \vec{\Pi} \\
\hline-\vec{\Pi}_{z} P \mathcal{C}_{\vec{\tau}} & -\vec{\Pi}_{z} \mathcal{C}_{\vec{\tau}} \pi & \mathbf{1}+\vec{\Pi}_{z} \mathbb{G}^{\vec{\Pi}}
\end{array}\right]
$$

The identity is seen by

$$
\begin{gathered}
{\left[\begin{array}{c|c|c|c|c}
\mathbf{1} & \mathcal{C}^{T} \pi & -\mathcal{C}_{-\vec{\tau}}^{T} P \vec{\Pi} \\
\hline \pi \mathcal{C} & \mathbf{1} & -\pi \mathcal{C}_{-\vec{\tau}}^{T} \vec{\Pi} \\
\hline-\vec{\Pi}_{z} P \mathcal{C}_{\vec{\tau}} & -\vec{\Pi}_{z} \mathcal{C}_{\vec{\tau}} \pi & \mathbf{1}+\vec{\Pi}_{z} \mathbb{G} \vec{\Pi}
\end{array}\right]\left[\begin{array}{c|c|c|}
\mathbf{1} & -\mathcal{C}^{T} \pi & \mathcal{C}_{-\vec{\tau}}^{T} P \vec{\Pi} \\
\hline 0 & \mathbf{1} & 0 \\
\hline 0 & 0 & \mathbf{1}
\end{array}\right]=} \\
{\left[\begin{array}{c|c|c}
\mathbf{1} & 0 & 0 \\
\hline \pi \mathcal{C} & \mathbf{1}-\pi \mathcal{C C}^{T} \pi & -\mathcal{C}_{-\vec{\tau}}^{T} \vec{\Pi}+\pi \mathcal{C C}_{-\vec{\tau}}^{T} P \vec{\Pi} \\
\hline-\vec{\Pi}_{z} P \mathcal{C}_{\vec{\tau}} & -\vec{\Pi}_{z} \mathcal{C}_{\vec{\tau}}+\vec{\Pi}_{z} P \mathcal{C}_{\vec{\tau}} \mathcal{C}^{T} \pi & \mathbf{1}+\vec{\Pi}_{z} \vec{G} \vec{\Pi}-\vec{\Pi}_{z} P \mathcal{C}_{\vec{\tau}} \mathcal{C}_{-\vec{\tau}}^{T} P \vec{\Pi}
\end{array}\right]}
\end{gathered}
$$

The operator $\mathbb{H}$ is read off $(3.22)$. Q.E.D

\subsection{From the Tacnode to the Pearcey process}

Given its origins in the Dyson Brownian diffusion, and by the same idea that led to considering the limit of the Pearcey to the Airy process, it is physically expected that the tacnode process should converge to the Pearcey process under a suitable rescaling sending the overlap $\sigma$ to $-\infty$, which amounts to "push" closer and closer the two sets of particles touching on the tacnode point. A rigorous approach based on the representation of the gap-probabilities in terms of a RiemannHilbert problem [7] suggests that this convergence occurs in the following asymptotic regime

$$
a_{t a c}=\frac{a_{P}}{(-8 \sigma)^{\frac{1}{8}}}, \quad \tau_{t a c}= \pm \sqrt{\frac{-\sigma}{2}}+\frac{\tau_{P}}{\left(-2^{7} \sigma\right)^{\frac{1}{4}}}, \quad \sigma \rightarrow-\infty
$$

in other words we have that

$$
\lim _{\sigma \rightarrow-\infty} F_{\operatorname{tac}}\left(\left[\frac{a_{P}}{(-8 \sigma)^{\frac{1}{8}}}, \frac{b_{P}}{(-8 \sigma)^{\frac{1}{8}}}\right], \pm \sqrt{\frac{-\sigma}{2}}+\frac{\tau_{P}}{\left(-2^{7} \sigma\right)^{\frac{1}{4}}}, \sigma\right)=F_{\mathrm{P}}\left(\left[a_{P}, b_{P}\right], \tau_{P}\right) .
$$

Indeed, modulo a multiplicative rescaling of the variables (but without changing the scale exponents) the formula above is proven in [13], Theorem 2.3 (more correctly, (3.25) is a direct consequence of the quoted theorem, since the uniform convergence of the kernels on compact sets implies the convergence of the Fredholm determinants). In [7] we will give another proof of (3.25), based on the nonlinear steepest descent analysis of a different Riemann-Hilbert problem giving directly the 
gap probability of the process rather than its kernel.

Here we show numerically the validity of (3.25) by computing the following two gap-probabilities:

$$
\begin{array}{r}
F_{\text {tac }}\left(\left[a_{\text {tac }}, b_{\text {tac }}\right] ; \tau_{\text {tac }}, \sigma\right):=\mathbb{P}_{\sigma}\left\{\mathcal{T}_{\sigma}\left(\tau_{\text {tac }}\right) \notin\left[a_{\text {tac }}, b_{\text {tac }}\right]\right\} \\
F_{\mathrm{P}}\left(\left[a_{P}, b_{P}\right], \tau_{\text {tac }}\right):=\mathbb{P}\left\{\mathcal{P}\left(\tau_{P}\right) \notin\left[-a_{P}, a_{P}\right]\right\}
\end{array}
$$

and verifying numerically the convergence. This has been done for $-\sigma$ up to 9 , after which our ${ }^{5}$ numerical implementation of the Fredholm determinant becomes too unstable, see Fig. 2. We have also verified in a numerical example the convergence of a two-time gap probability, see Fig. 3.

\subsection{From the tacnode to the Airy process}

There are two types of regimes in which the gap probability for the tacnode process "degenerates" into the Airy one:

1. large separation $\sigma \rightarrow+\infty$; physically it corresponds to pull far apart the two sets of Brownian particles touching on the tacnode point.

2. large time $\tau \rightarrow \pm \infty$; it corresponds to move far away from the singular point along the boundary of the space-time region swept out by the particles.

Let us for simplicity describe the one-interval and one-time case. Keeping the overlap $\sigma$ fixed, we will have that

$$
\lim _{\tau \rightarrow \infty} F_{\text {tac }}\left(\left[a-\sigma-\tau^{2}, b-\sigma-\tau^{2}\right], \tau, \sigma\right)=F_{2}([a, b])
$$

while, keeping $\tau$ fixed, in a complete analogous way we obtain

$$
\lim _{\sigma \rightarrow \infty} F_{\mathrm{tac}}\left(\left[a-\sigma-\tau^{2}, b-\sigma-\tau^{2}\right], \tau, \sigma\right)=F_{2}([a, b]) .
$$

This convergence is simple to see by inspecting directly the kernel of the (extended) tacnode process (3.1) because the term involving the resolvent of the Airy kernel tends to zero, uniformly over compact sets of the translated spatial variables $x-\sigma-\tau^{2}$. Here we will not study the rate of convergence in formulas (3.28), (3.29). We simply point out that, if we knew some equation for the tacnode gap probability, we could perform a similar analysis to the one in [1]. The extension to multi-time case (and multi-intervals), though more cumbersome, does not present any additional difficulty.

A perhaps more interesting situation, less obvious from the formula (3.1) but equally natural from the "physical" setting, is the one in which the tacnode process degenerates into a couple of Tracy-Widom distributions, in analogy with the Pearcey-to-Airy transition (2.8) proved in [6]. In this case, roughly speaking, half of the space variables (endpoints of the gaps) moves far away from the tacnode following the left branch of the boundary of the space-time region swept by the particles, and the other half goes in the opposite direction. The simplest instance of this degeneration is the case of one-time and one-interval $E=\left[a-\sigma-\tau^{2},-b+\sigma+\tau^{2}\right]$ :

$$
\lim _{\sigma \rightarrow \infty} F_{\text {tac }}\left(\left[a-\sigma-\tau^{2},-b+\sigma+\tau^{2}\right], \tau, \sigma\right)=F_{2}([a, \infty)) F_{2}([b, \infty)) .
$$

Similarly, the limit (3.30) holds also for $\tau \rightarrow \pm \infty$ and $\sigma$ fixed. The formula (3.30), as well as its generalization to the multi-interval case, will be proven in [7], numerically these regimes are illustrated in Figure 4.

\footnotetext{
${ }^{5}$ None of the author specializes in numerical computations, and hence we have implemented the ideas of [8] in its simplest form. See Appendix.
} 


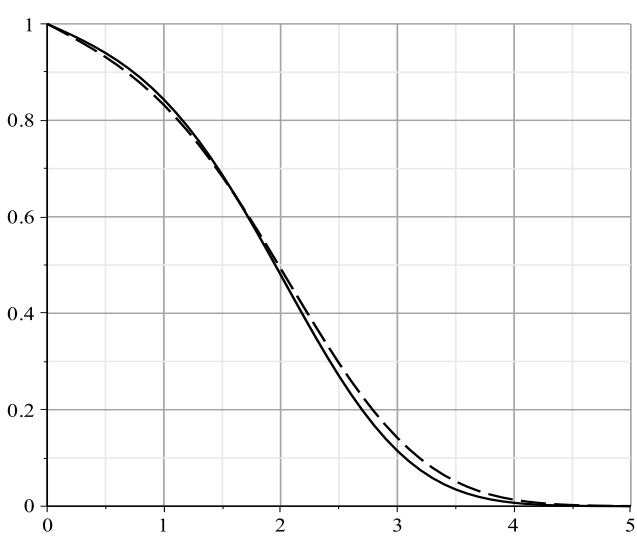

The plot of $F_{t a c}$ (solid) and $F_{\text {Pearcey }}\left(\right.$ dash) with $\sigma=-1$ and $\tau_{P}=1$

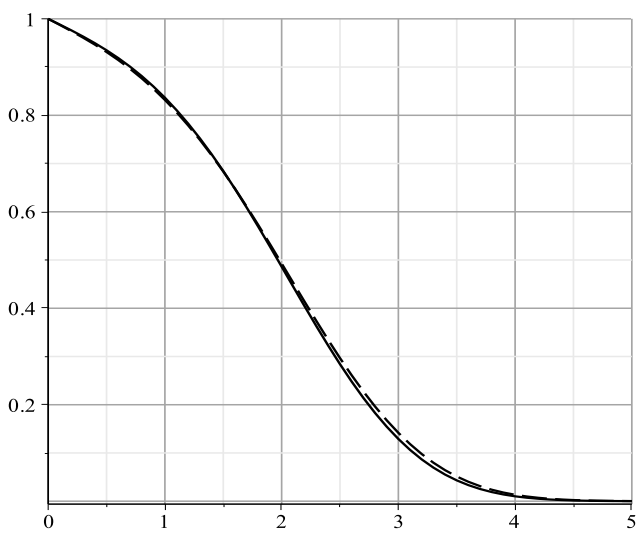

The plot of $F_{\text {tac }}$ (solid) and $F_{\text {Pearcey }}$ (dash) with $\sigma=-3$ and $\tau_{P}=0$

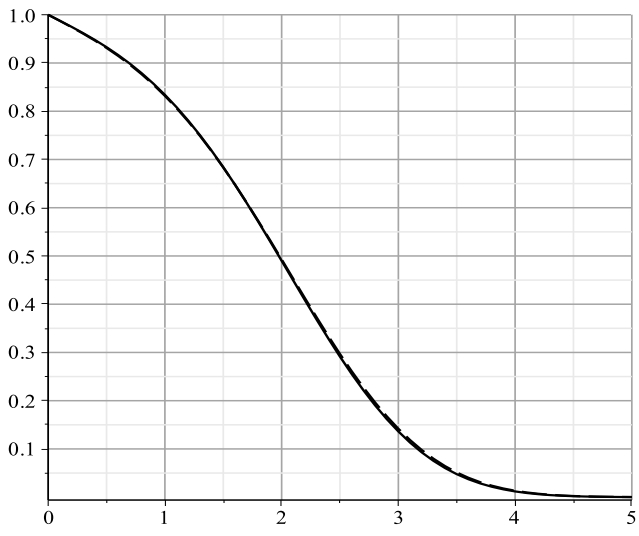

The plot of $F_{\text {tac }}$ (solid) and $F_{\text {Pearcey }}$ (dash) with $\sigma=-9$ and $\tau_{P}=1$

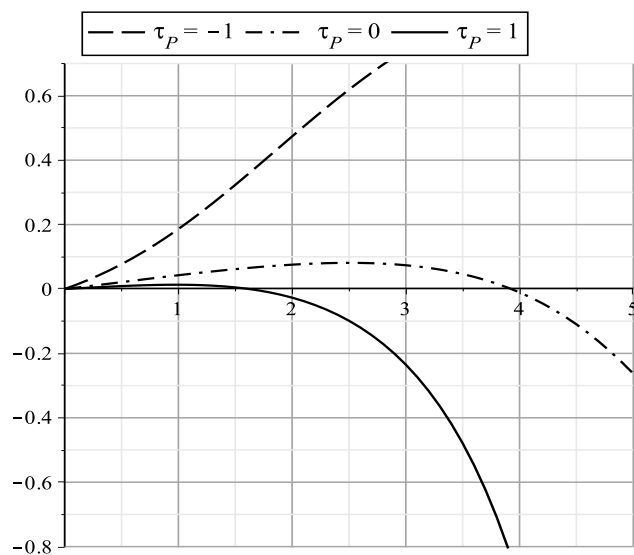

The relative error of $F_{\text {tac }}$ and $F_{\text {Pearcey }}$ with $\sigma=-1$

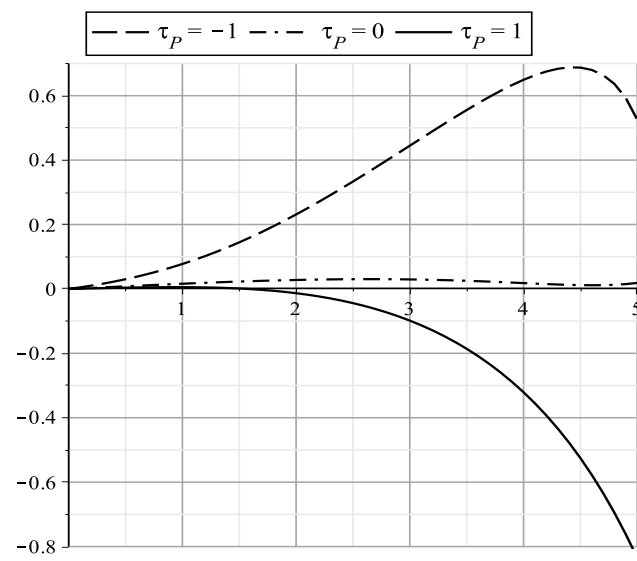

The relative error of $F_{t a c}$ and $F_{\text {Pearcey }}$ with $\sigma=-3$

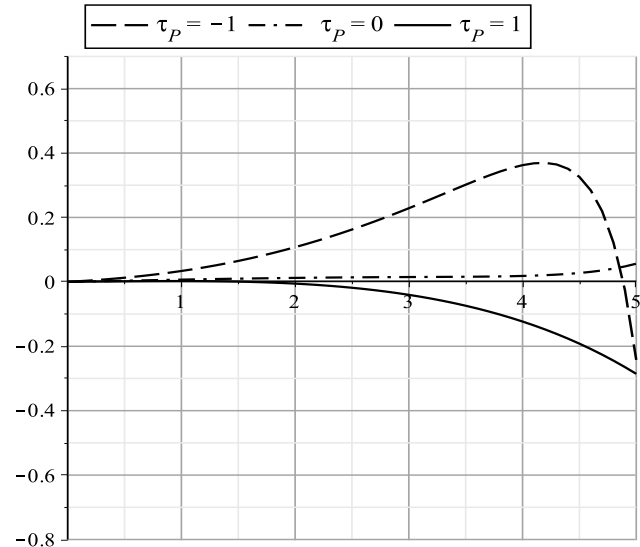

The relative error of $F_{t a c}$ and $F_{\text {Pearcey }}$ with $\sigma=-9$

Figure 2: The graphs of $F_{\mathrm{tac}}\left(\left[-a_{t a c}, a_{t a c}\right], \tau_{t a c} ; \sigma\right)$ and $F_{\mathrm{P}}\left(\left[-a_{P}, a_{P}\right] ; \tau_{P}\right)$ with the parameters related by (3.24). 

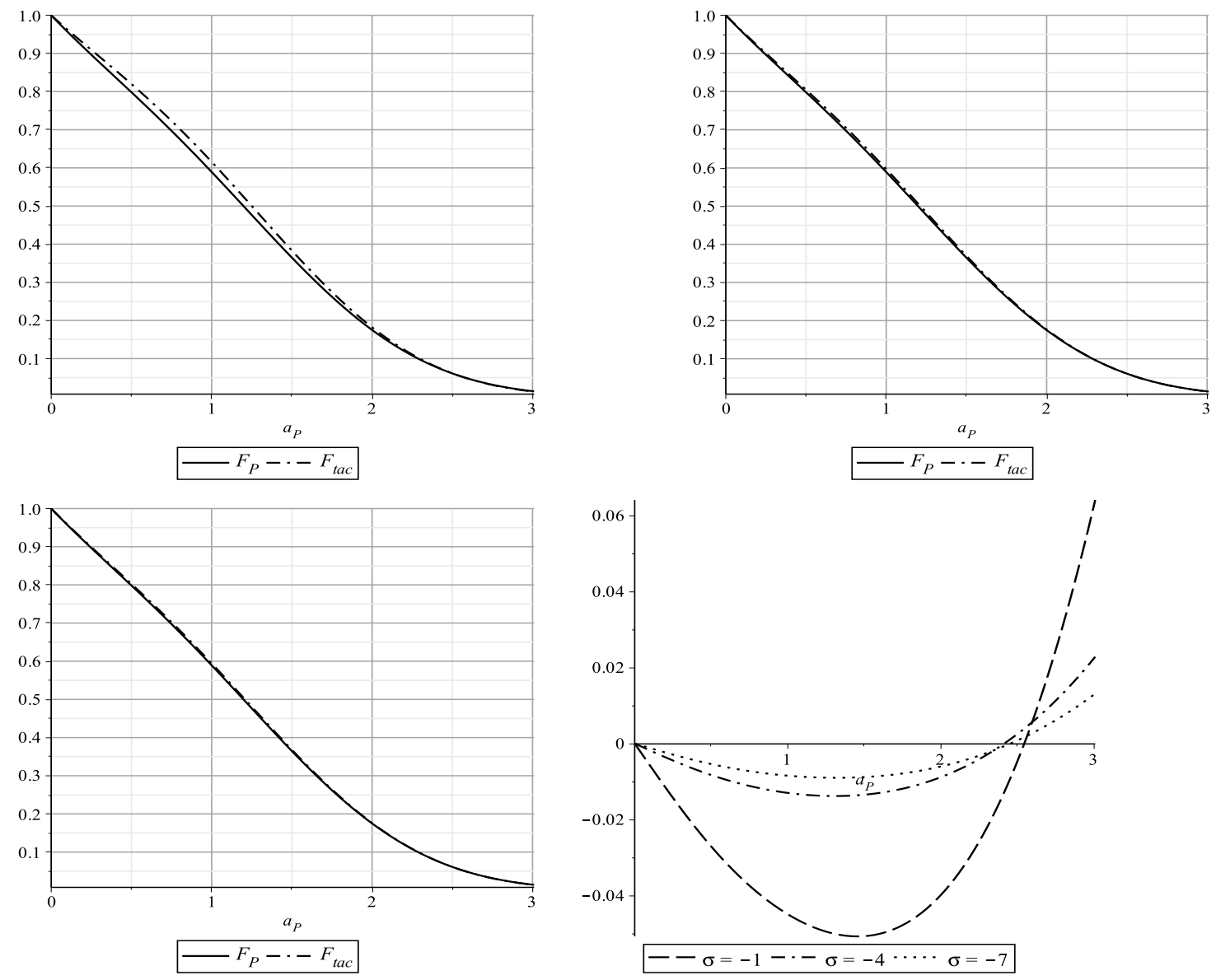

Figure 3: The multi-time case. Here the graphs of $F_{\text {tac }}\left(\left\{\left[-a_{t a c}, a_{t a c}\right] ; \tau_{t a c, 1},\left[-a_{t a c}, a_{t a c}\right] ; \tau_{t a c, 2}\right\} ; \sigma\right)$ and $F_{\mathrm{P}}\left(\left\{\left[-a_{P}, a_{P}\right] ; \tau_{P, 1},\left[-a_{P}, a_{P}\right] ; \tau_{P, 2}\right\}\right)$ are shown with the parameters related by $(3.24)$ and $\tau_{P, 1}=0, \tau_{P, 2}=1$. The last graph is the plot of the relative discrepancy between $F_{\mathrm{P}}$ and $F_{\mathrm{tac}}$. 

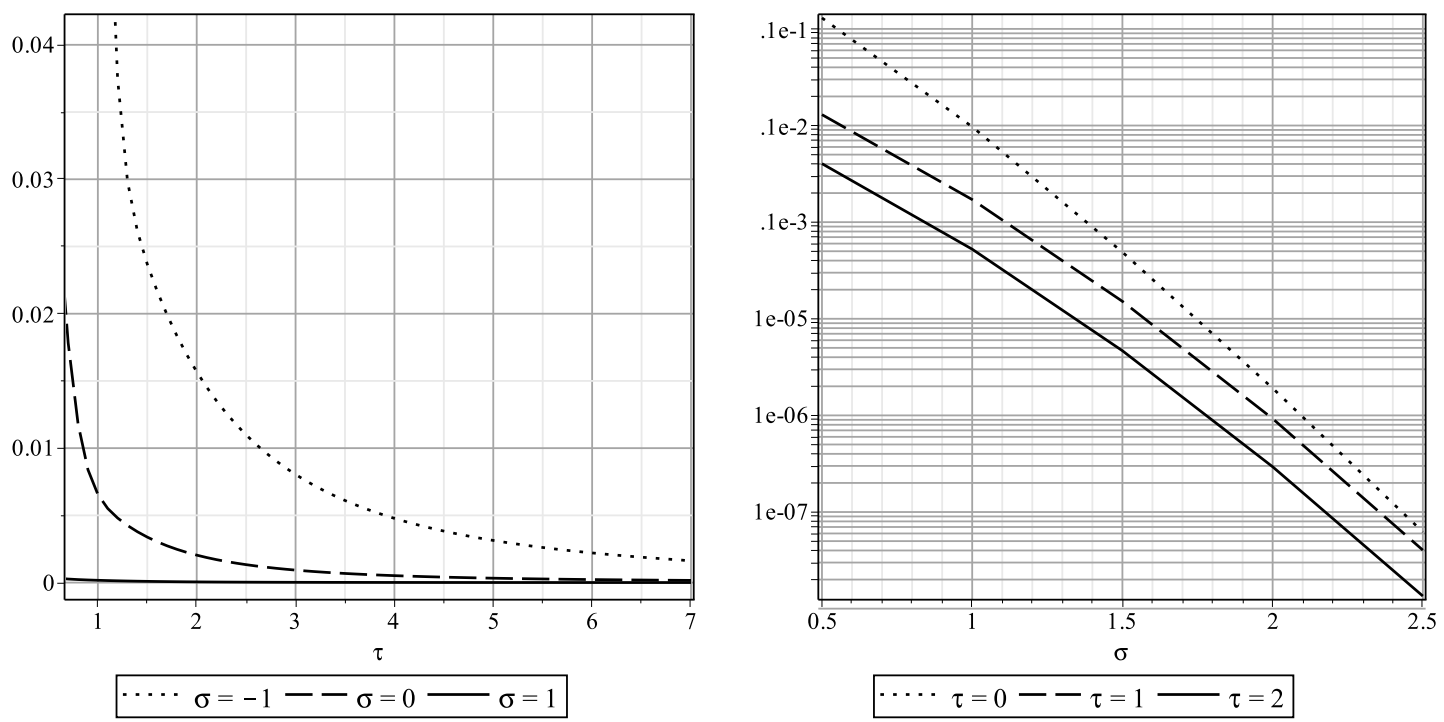

Figure 4: The relative values $1-\frac{F_{\text {tac }}\left(\left[a_{t a c}, b_{t a c}\right] ; \tau_{t a c}, \sigma\right)}{F_{2}(a) F_{2}(b)}$ with $a_{t a c}=a-\sigma-\tau_{t a c}^{2}, b_{t a c}=b+\sigma+\tau_{t a c}^{2}$, plotted against $\tau_{\text {tac }}$ (left) and $\sigma$ (right), showing the convergence of the tacnode gap probability to the product of two Tracy-Widom distributions. Here, by the way of example, $a=-0.3, b=0.5$. The convergence as $\sigma \rightarrow \infty$ is clearly exponential (note that the graph on the right is on a semilog coordinate system).

\section{Acknowledgements}

The work of M.B. is partially supported by the Natural Sciences and Engineering Research Council of Canada (NSERC) and the work of M.C. is partially supported by the ANR project DIADEMS. M.C. thanks the UMI CRM/CNRS for the hospitality and the financial support during his stay in Montreal, and he is also grateful to A.Hardy and L.Chaumont for very useful suggestions and discussions.

\section{A Conditioned processes}

Consider a determinantal random point field (process, DRPF) $\mathcal{R}$ on the configuration space $\mathcal{X}$, with kernel $K: \mathcal{X} \times \mathcal{X} \rightarrow \mathbb{R}$; we assume that $\mathcal{X}$ is endowed with a measure $\mathrm{d} \mu$ and $K$ can be interpreted as an operator in $L^{2}(\mathcal{X}, \mathrm{d} \mu)$. The knowledge of the occupation probabilities of any point process is equivalent to the knowledge of their correlation functions by means of the generating functions of the occupation numbers:

$$
F(E ; \vec{z}):=\left\langle\prod_{j=1}^{k}\left(z_{j}\right)_{E_{j}}^{\sharp}\right\rangle=\sum_{\vec{\ell} \in \mathbb{N}^{k}} \mathbb{P}\left\{\sharp_{E_{j}}=\ell_{j}, \quad j=1, \ldots k\right\} \prod_{j=1}^{k} z_{j}^{\ell_{j}}
$$

where we denote with $E:=E_{1} \sqcup \ldots \sqcup E_{r}$ an arbitrary disjoint union of intervals. Denoting the indicator function of a set by $\Pi_{E}$, also thought of as orthogonal projection, the above generating 
function is computed as a Fredholm determinant (see [21] for details)

$$
F(E ; \vec{z})=\operatorname{det}\left[\mathbf{1}_{\mathcal{X}}-\left(\sum_{j=1}^{k}\left(1-z_{j}\right) \Pi_{E_{j}}\right) K \Pi_{E}\right] .
$$

Let $\mathcal{A} \subset \mathcal{X}$. We want to study the conditional process (field) $\mathcal{R}_{\mathcal{A}}$ on $\mathcal{Y}:=\mathcal{X} \backslash \mathcal{A}$ as the process "conditioned to have no points in $\mathcal{A}$ ".

Remark A.1 For any $\mathcal{A} \subset \mathcal{X}$ denote by $\sharp_{\mathcal{A}}$ the integer-valued random variable counting the points of a configuration that belong to $\mathcal{A}$. Let $E=E_{1} \sqcup \ldots \sqcup E_{k} \subset \mathcal{Y}$ : then the conditional probabilities of occupation numbers $\mathbb{P}_{\mathcal{A}}$ are given as usual as

$$
\mathbb{P}_{\mathcal{A}}\left\{\sharp_{E_{j}}=k_{j}, \quad j=1, \ldots k\right\}:=\frac{\mathbb{P}\left\{\sharp_{E_{j}}=k_{j}, j=1, \ldots k, \sharp_{\mathcal{A}}=0\right\}}{\mathbb{P}\left\{\sharp_{\mathcal{A}}=0\right\}}
$$

On the other hand, according to the general setup for determinantal random point fields, the conditional process is completely determined by all possible generating functions for the occupation numbers, denoted below with $F_{\mathcal{A}}(E ; \vec{z})$ :

$$
\begin{array}{r}
F_{\mathcal{A}}(E ; \vec{z})=\sum_{\vec{\ell} \in \mathbb{N}^{k}} \mathbb{P}_{\mathcal{A}}\left\{\sharp_{E_{j}}=\ell_{j}, \quad j=1, \ldots k\right\} \prod_{j=1}^{k} z_{j}^{\ell_{j}}= \\
=\sum_{\vec{\ell} \in \mathbb{N}^{k}} \frac{\mathbb{P}\left\{\sharp_{E_{j}}=k_{j}, \quad j=1, \ldots k, \sharp \mathcal{A}=0\right\}}{\mathbb{P}\left\{\sharp_{\mathcal{A}}=0\right\}} \prod_{j=1}^{k} z_{j}^{\ell_{j}}=\frac{F(E \sqcup \mathcal{A} ;(\vec{z}, 0))}{F(\mathcal{A} ; 0)}
\end{array}
$$

where the denominator of the last formula should be understood as

$$
F(E \sqcup \mathcal{A} ;(\vec{z}, 0))=\operatorname{det}\left[\mathbf{1}_{\mathcal{X}}-\left(\left(\sum_{j=1}^{k}\left(1-z_{j}\right) \Pi_{E_{j}}\right)+\Pi_{\mathcal{A}}\right) K \Pi_{E \sqcup \mathcal{A}}\right] .
$$

Note, in particular, that both the numerator and denominator are Fredholm determinants of kernels, exactly as in Section 3 above,Theorem 3.2. Below we observe that, given a determinantal process, the associated conditional processes are also determinantal themselves (see also [22] and [17]).

Proposition A.1 Suppose you are given a determinantal random point field $\mathcal{R}$ on $\mathcal{X}$ with kernel $K$, and let $\mathcal{A} \subseteq \mathcal{X}$ an arbitrary measurable subset of the state space. Then the associated conditional process $\mathcal{R}_{\mathcal{A}}$ on $\mathcal{Y}:=\mathcal{X} \backslash \mathcal{A}$ is also determinantal with kernel

$$
K_{\mathcal{A}}: \mathcal{Y} \times \mathcal{Y} \rightarrow \mathbb{R}
$$

given by

$$
K_{\mathcal{A}}\left(y_{1}, y_{2}\right)=K\left(y_{1}, y_{2}\right)+\int_{\mathcal{A}} K\left(y_{1}, a_{1}\right)\left(\mathbf{1}_{\mathcal{A}}-\left.K\right|_{\mathcal{A}}\right)^{-1}\left(a_{1}, a_{2}\right) K\left(a_{2}, y_{2}\right) \mathrm{d} \mu\left(a_{1}\right) \mathrm{d} \mu\left(a_{2}\right)
$$

or, in operator notation,

$$
K_{\mathcal{A}}:=K+K \Pi_{A}\left(\mathbf{1}_{\mathcal{A}}-\left.K\right|_{\mathcal{A}}\right)^{-1} \Pi_{\mathcal{A}} K
$$

Here $\Pi_{\mathcal{A}}$ denotes the projection onto $L^{2}(\mathcal{A}) \subset L^{2}(\mathcal{X})$. 
Proof The proof relies on the computation of all possible generating functions. We start the numerator of the right side of (1.5): we realize the integral operator defined by $K$ restricted to $E \sqcup \mathcal{A}=E_{1} \sqcup \ldots \sqcup E_{k} \sqcup \mathcal{A}$ as an operator on $L^{2}\left(E_{1}\right) \oplus \ldots \oplus L^{2}\left(E_{k}\right) \oplus L^{2}(\mathcal{A})$. With obvious block-matrix notation and denoting $\lambda_{j}=1-z_{j}$, we then have the specialization of the general determinantal formula $(1.2)$ is

$$
F(E \sqcup \mathcal{A} ;(\vec{z}, 0))=\operatorname{det}\left[\begin{array}{c|c|c|c}
\mathbf{1}_{\mathcal{A}}-K_{\mathcal{A} \mathcal{A}} & -K_{\mathcal{A} E_{1}} & \ldots & -K_{\mathcal{A} E_{k}} \\
-\lambda_{1} K_{E_{1} \mathcal{A}} & \mathbf{1}_{E_{1}}-\lambda_{1} K_{E_{1} E_{1}} & \ldots & -\lambda_{1} K_{E_{1} E_{k}} \\
\vdots & & & \\
-\lambda_{k} K_{E_{k} \mathcal{A}} & -\lambda_{k} K_{E_{k} E_{1}} & \ldots & \mathbf{1}_{E_{k}}-\lambda_{k} K_{E_{k} E_{k}}
\end{array}\right]
$$

Then the computation goes as follows (we denote for brevity $R:=\Pi_{\mathcal{A}}\left(\mathbf{1}_{\mathcal{A}}-K_{\mathcal{A A}}\right)^{-1} \Pi_{\mathcal{A}}$

$$
\begin{aligned}
& \frac{1}{\operatorname{det}\left(\mathbf{1}_{A}-K_{\mathcal{A A}}\right)} \operatorname{det}\left[\begin{array}{c|ccc}
\mathbf{1}_{\mathcal{A}}-K_{\mathcal{A} \mathcal{A}} & -K_{\mathcal{A} E_{1}} & \ldots & -K_{\mathcal{A} E_{k}} \\
\hline-\lambda_{1} K_{E_{1} \mathcal{A}} & \mathbf{1}_{E_{1}}-\lambda_{1} K_{E_{1} E_{1}} & \ldots & -\lambda_{1} K_{E_{1} E_{k}} \\
\vdots & & & \\
-\lambda_{k} K_{E_{k} \mathcal{A}} & -\lambda_{k} K_{E_{k} E_{1}} & \ldots & \mathbf{1}_{E_{k}}-\lambda_{k} K_{E_{k} E_{k}}
\end{array}\right]= \\
& \operatorname{det}\left[\begin{array}{c|ccc}
\mathbf{1}_{\mathcal{A}} & -R K_{\mathcal{A} E_{1}} & \ldots & -R K_{\mathcal{A} E_{k}} \\
\hline-\lambda_{1} K_{E_{1} \mathcal{A}} & \mathbf{1}_{E_{1}}-\lambda_{1} K_{E_{1} E_{1}} & \ldots & -\lambda_{1} K_{E_{1} E_{k}} \\
\vdots & & & \\
-\lambda_{k} K_{E_{k} \mathcal{A}} & -\lambda_{k} K_{E_{k} E_{1}} & \ldots & \mathbf{1}_{E_{k}}-\lambda_{k} K_{E_{k} E_{k}}
\end{array}\right]= \\
& \operatorname{det}\left[\begin{array}{c|ccc}
\mathbf{1}_{\mathcal{A}} & 0 & \ldots & 0 \\
\hline \lambda_{1} K_{E_{1} \mathcal{A}} & \mathbf{1}_{E_{1}} & \ldots & 0 \\
\vdots & & & \\
\lambda_{k} K_{E_{k} \mathcal{A}} & 0 & \ldots & \mathbf{1}_{E_{k}}
\end{array}\right]\left[\begin{array}{c|ccc}
\mathbf{1}_{\mathcal{A}} & -R K_{\mathcal{A} E_{1}} & \ldots & -R K_{\mathcal{A} E_{k}} \\
\hline-\lambda_{1} K_{E_{1} \mathcal{A}} & \mathbf{1}_{E_{1}}-\lambda_{1} K_{E_{1} E_{1}} & \ldots & -\lambda_{1} K_{E_{1} E_{k}} \\
\vdots & & & \\
-\lambda_{k} K_{E_{k} \mathcal{A}} & -\lambda_{k} K_{E_{k} E_{1}} & \ldots & \mathbf{1}_{E_{k}}-\lambda_{k} K_{E_{k} E_{k}}
\end{array}\right]= \\
& =\operatorname{det}\left[\begin{array}{c|ccc}
\mathbf{1}_{\mathcal{A}} & -R K_{\mathcal{A} E_{1}} & \ldots & -R K_{\mathcal{A} E_{k}} \\
\hline 0 & \mathbf{1}_{E_{1}}-\lambda_{1}\left(K_{E_{1} E_{1}}+K_{E_{1} A} R K_{\mathcal{A} E_{1}}\right) & \ldots & -\lambda_{1}\left(K_{E_{1} E_{k}}+K_{E_{1} A} R K_{\mathcal{A} E_{k}}\right) \\
\vdots & & & \\
0 & -\lambda_{1}\left(K_{E_{k} E_{1}}+K_{E_{k} \mathcal{A}} R K_{\mathcal{A} E_{1}}\right) & \ldots & \mathbf{1}_{E_{k}}-\lambda_{1}\left(K_{E_{k} E_{k}}+K_{E_{k} A} R K_{\mathcal{A} E_{k}}\right)
\end{array}\right]= \\
& \operatorname{det}\left[\begin{array}{ccc}
\mathbf{1}_{E_{1}}-\lambda_{1}\left(K_{E_{1} E_{1}}+K_{E_{1} \mathcal{A}} R K_{\mathcal{A} E_{1}}\right) & \ldots & -\lambda_{1}\left(K_{E_{1} E_{k}}+K_{E_{1} \mathcal{A}} R K_{\mathcal{A} E_{k}}\right) \\
\vdots & & \\
-\lambda_{1}\left(K_{E_{k} E_{1}}+K_{E_{k} \mathcal{A}} R K_{\mathcal{A} E_{1}}\right) & \ldots & \mathbf{1}_{E_{k}}-\lambda_{1}\left(K_{E_{k} E_{k}}+K_{E_{k} A} R K_{\mathcal{A} E_{k}}\right)
\end{array}\right]
\end{aligned}
$$

The first determinant, $\operatorname{det}\left(\mathbf{1}_{\mathcal{A}}-K_{\mathcal{A A}}\right)=F_{\mathcal{A}}(0)$ is the probability of finding no points in $\mathcal{A}$ and the last determinant is precisely the generating function of occupation numbers for the restricted process $K_{\mathcal{A}}$, which completes the proof. Q.E.D

\section{References}

[1] M. Adler, M. Cafasso, and P. van Moerbeke. From the Pearcey to the Airy process. Electron. J. Probab., 16(36):1048-1064, 2011. 
[2] M. Adler, P.L. Ferrari, and P. van Moerbeke. Non-intersecting random walks in the neighborhood of a symmetric tacnode. arXiv:1007.1163, 2010.

[3] Mark Adler, Kurt Johansson, and Pierre van Moerbeke. Double aztec diamonds and the tacnode process. arXiv:1112.5532, 2011.

[4] Gernot Akemann, Jinho Baik, and Philippe Di Francesco. The Oxford handbook of random matrix theory. Oxford University Press, Oxford, 2011.

[5] M. Bertola and M. Cafasso. Riemann-Hilbert approach to multi-time processes; the Airy and the Pearcey cases. Physica D, 241(23-24):2237-2245, 2012.

[6] M. Bertola and M. Cafasso. The Riemann-Hilbert approach to the transition between the gap probabilities from the Pearcey to the Airy process. International Mathematics Research Notices, 2012(7):1519-1568, 2012.

[7] M. Bertola M. Cafasso and M. Girotti. The tacnode process, isomonodromic tau functions and applications. In preparation, 2013.

[8] Folkmar Bornemann. On the numerical evaluation of Fredholm determinants. Math. Comp., 79(270):871-915, 2010.

[9] Steven Delvaux. The tacnode kernel: equality of Riemann-Hilbert and Airy resolvent formulas. arXiv:1211.4845, 2012.

[10] Steven Delvaux, Arno B. J. Kuijlaars, and Lun Zhang. Critical behavior of nonintersecting Brownian motions at a tacnode. Comm. Pure Appl. Math., 64(10):1305-1383, 2011.

[11] Freeman J. Dyson. A Brownian-motion model for the eigenvalues of a random matrix. $J$. Mathematical Phys., 3:1191-1198, 1962.

[12] Patrik L. Ferrari and Bálint Veto. Non-colliding Brownian bridges and the asymmetric tacnode process. Electron. J. Probab., 17(44), 2012.

[13] Dries Geudens and Lun Zhang. Transitions between critical kernels: from the tacnode kernel and critical kernel in the two-matrix model to the Pearcey kernel. arXiv:1208.0762, 2012.

[14] Kurt Johansson. Discrete polynuclear growth and determinantal processes. Comm. Math. Phys., 242(1-2):277-329, 2003.

[15] Kurt Johansson. The arctic circle boundary and the Airy process. Ann. Probab., 33(1):1-30, 2005.

[16] Kurt Johansson. Non-colliding Brownian motions and the extended tacnode process. arXiv:1105.4027, 2011. 
[17] R. Lyons. Determinantal probability measures. Publ. Math. Inst. Hautes Études Sci., no. 98, 167-212, 2003.

[18] Andrei Okounkov and Nicolai Reshetikhin. Random skew plane partitions and the Pearcey process. Comm. Math. Phys., 269(3):571-609, 2007.

[19] Michael Prähofer and Herbert Spohn. Scale invariance of the PNG droplet and the Airy process. J. Statist. Phys., 108(5-6):1071-1106, 2002.

[20] Barry Simon. Trace ideals and their applications, volume 120 of Mathematical Surveys and Monographs. American Mathematical Society, Providence, RI, second edition, 2005.

[21] A. Soshnikov. Determinantal random point fields. Uspekhi Mat. Nauk, 55(5(335)):107-160, 2000.

[22] T. Tao. The asymptotic distribution of a single eigenvalue gap of a Wigner matrix. Probab. Theory Relat. Fields, DOI 10.1007/s00440-012-0450-3.

[23] C. A. Tracy and H. Widom. Level-spacing distributions and the Airy kernel. Comm. Math. Phys., 159(1):151-174, 1994.

[24] Craig A. Tracy and Harold Widom. The Pearcey process. Comm. Math. Phys., 263(2):381-400, 2006. 